\title{
Study of Flow Induced Noise in Vertical Inline Pump Using Lighthill Analogy
}

\author{
Stephen Christopher ${ }^{1,2 *}$, Shouqi Yuan ${ }^{2}$, Ji Pei $^{2}$, G Xing Cheng ${ }^{2}$, and Wang Yiyun ${ }^{2}$ \\ ${ }^{1}$ Department of Mechanical Engineering, Saveetha School of Engineering, SIMATS, Chennai 602 105, Tamil Nadu, India \\ ${ }^{2}$ National Research Center of Pumps, Jiangsu University, Zhenjiang 212 013, Jiangsu Province, China
}

\begin{abstract}
The pump user demands for specification along with noise and vibration magnitude for extreme operating conditions apart from best efficiency flow. In order to predict the noise level in pumps, the entire unit of pump has to be considered for acoustic evaluation. Hence, the full three dimensional model of vertical inline pump which consists of inlet pipe, impeller, and volute casing is considered for detailing the flow induced noise using Lighthill analogy. The performance characteristics of pump are well matched between experimental and computational results and the overall sound power level at best efficiency flow using computation was compared with empirical relations and found to be agreed well. The streamline patterns along the inlet pipe shows the effects of recirculation and return flow at partload condition and confirm that the utilization of flow passage is not well streamlined even for nominal flow rate. Further, the detailed study of inlet pipe cross sections reveals the nature of different noise sources such as dipole and quadrupole sound levels along with Proudman source power level. The intensity of turbulence and vortices in flow passage is predicted well using quadrupole source and Proudman sound power levels.
\end{abstract}

\section{Introduction}

Many researchers have identified the various sources of noise inside the pump. The occurrence of noise is mainly due to the interaction between two domains such as impeller and volute, in addition, noises are due to the flow nature inside the pump. The details of flow physics and their sources of noise are discussed by Gülich [1]. A centrifugal pump produces hydraulic noise corresponds to vane passing frequency and its harmonics and at shaft frequency with related harmonics [2]. Further it was confirmed that the main source of noise generation from pumps is from vane tongue interaction [3,4]. The detection of noise radiation from the two dimensional model of centrifugal pump was analyzed by Langthjem et al. [5] using Lighthill acoustic analogy and concludes that there was a good agreement between numerical and experimental readings. Jiang et al. [6] studied the feasible method to couple fluid structure interaction with flow induced noise in turbomachines using Large Eddy Simulation (LES) based CFD and FEM. The efficiency of noise radiation from a centrifugal pump for different blades shapes was determined by Huang [7] using numerical simulation hybrid with Ffowcs WilliamsHawkings (FW-H) acoustic model.

The three dimensional unsteady flow was simulated with $k-\varepsilon$ turbulence model and coupled with LMS Virtual Lab to estimate the sound field inside the pump and the results concluded that dipole source was the main cause for flow induced noise [8]. The noise generation by the blades as well as from the volute of centrifugal pump was captured using FW-H model and found to be well agreed with experimental findings [9].
The gap in literature is that, most of the researcher concentrated on impeller and volute but not on the suction pipe of centrifugal pump or the inlet pipe of vertical inline pump. Hence in this work, commercial CFD code called ANSYS CFX 17.0 is used for determination of flow induced noise in all components of vertical inline pump using Lighthill analogy. The relation between dipole, quadrupole sources of noise as well as the Proudman sound power using Lighthill analogy are analyzed for inlet pipe for various flow conditions.

\section{Numerical Approach}

\subsection{Lighthill Analogy}

The analogy for prediction of sound from the flow field was detailed by Lighthill [10] from the equations of continuity and momentum and hence deduced to wave equation. The flow noise was predicted successfully for implementation of acoustic analogy derived by Ffowcs Williams and Hawkings to all fluid machinery applications [11]. A generalized version of the Lighthill equation that represents the noise sources is given by Williams, et al. [12] as follows:

$$
\underbrace{\frac{\partial^{2} \rho^{\prime}}{\partial t^{2}}-c_{s}{ }^{2} \frac{\partial^{2} \rho^{\prime}}{\partial x_{i}^{2}}}_{A}=\underbrace{\frac{\partial}{\partial t}\left\{\rho v_{n} \delta(f)\right\}}_{B}-\underbrace{\frac{\partial}{\partial x_{i}}\left\{\tau_{i j} \delta(f)\right\}}_{C}+\underbrace{\frac{\partial^{2} T_{i j}}{\partial x_{i} \partial x_{j}}}_{D}(1)
$$

Eq. (1) represents the wave equation (A) with three sources of noise term in flow, they are monopole (B), dipole (C), and quadrupole (D). The mass flow 
fluctuation is related to monopole source and it is called as thickness noise due to non uniform flow, dipole is the pressure fluctuation in flow field (or loading noise). Both monopole and dipole are the surface source terms, whereas quadrupole is volume source term. Quadrupole is mainly because of turbulence or vortices or in other term fluctuating shear stress in the flow domain. But, for low Mach number applications, direct noise emission from quadrupole source is of no practical importance in water [13]. Conversely, Hanson \& Fink [14] proved that the substantial agreement between test data was obtained only by including quadrupole source term. Lighthill stress tensor $\left(T_{i j}\right)$ is defined as the summation of momentum flux tensor $\left(\rho v_{i} v_{j}\right)$ and fluid stress tensor $\left(\tau_{i j}\right)$ and subtracted by stress in the uniform acoustic medium at rest $\left(c_{s}^{2} \rho^{\prime} \delta_{i j}\right)$.

Proudman [15] derived a method to extract the noise radiation from isotropic turbulence at low Mach number with high Reynolds number from Lighthill theory for complete flow field. The total acoustic power $\left(P_{A}\right)$ which is the function of time averaged kinetic energy and its dissipation rate per unit volume is given in Eq. (2).

$$
P_{A}=\alpha \rho \varepsilon\left(\frac{\sqrt{2 k}}{c_{s}}\right)^{5}
$$

Sarkar and Kussaini [16] calibrated the constant $(\alpha)$ to determine sound power level which is also known as broadband noise source in the flow domain. Eq. (2) is rewritten with reference to acoustic reference power in $\mathrm{dB}$ to determine Proudman sound power level (Psp). The fluctuation of near field pressure and velocity plays a vital role in determination of far field noise levels. Hence the present work is discussed based on near field parameters distribution throughout the flow domains of vertical inline pump.

\subsection{Flow Domain and Discretization}

The test pump was designed for head of $20 \mathrm{~m}$ and for flow rate of $0.0014 \mathrm{~m}^{3} / \mathrm{s}$ at $2910 \mathrm{rpm}$. The resultant impeller is of double curvature radial flow with six vanes. The diameters of suction and delivery pipes are same, which is about $80 \mathrm{~mm}$ with the length of 10 times the pipe diameter to have uniform flow at the entry of inlet pipe and to predict the exact flow nature at the exit of diffuser. Figure 1 shows the mesh of low specific speed pump $\left(n_{s}=36\right)$ of inlet pipe, impeller, and volute casing.

Using ICEM CFD 17.0, the flow models was discretized with structural mesh by proper consideration of $y^{+}$near the wall and at critical surfaces of vertical inline pump which was between 5 to 10 and maximum of 80 was adapted for other surfaces of domains. Lakshminarayana [17] stated that the solution from flow equations are strongly depends on the parameters during setup conditions such as grid size, turbulence model, time step, boundary conditions and so on. Based on the grid sensitivity studies, the following number of nodes is used for simulation they are 0.39 millions, 0.42 millions, and 0.5 millions for inlet pipe, impeller, and volute casing with radial discharge respectively and 1.56 millions is for suction and delivery pipes. The head coefficient and efficiency discrepancy between the maximum number of nodes and the optimized nodes are $0.4 \%$ and $0.3 \%$ respectively.

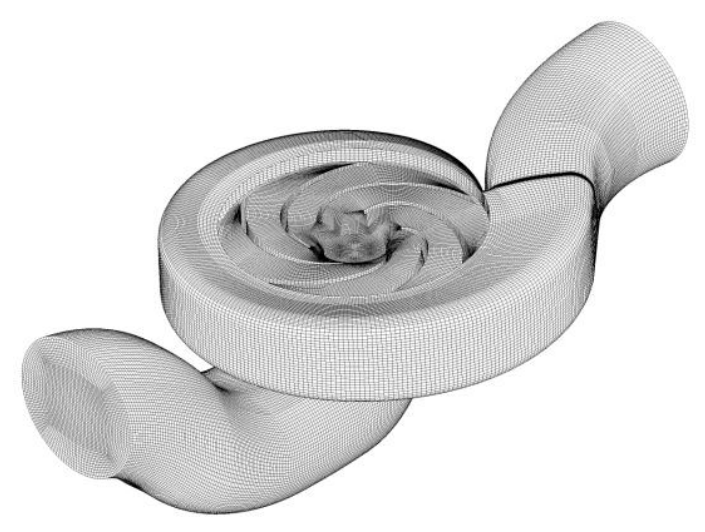

Fig. 1. Mesh of vertical inline pump

\subsection{Setup Conditions for Simulations}

The steady state simulations were performed from $50 \%$ to $150 \%$ of nominal flow rate $(\mathrm{Qn})$ in the step of $10 \%$, whereas for transient case, $50 \%, 80 \%, 100 \%, 120 \%$, and $150 \%$ of Qn were solved. The total pressure (stable) and mass flow rate are given at the inlet of suction and outlet of delivery pipes respectively, which was taken from the real working condition of pump. The results from steady state were used as initial guess for transient analysis with time step of $1.72 \times 10-4 \mathrm{~s}$ for the impeller rotation of 3 deg. Around 1.5 times the impeller rotation was simulated to extract the results for acoustic studies with water as working fluid at the reference pressure of $101.325 \mathrm{kPa}$ and at temperature $25^{\circ} \mathrm{C}$. The interface between stationary and rotating domains was set to 'transient rotor stator' for transient case while for steady case it was 'frozen rotor'. Shear stress transport (SST) model was considered for identification of turbulence in flow domain especially in near wall region and for determination of flow separation [18]. The computed results were converged, when the residual value is lower than or equal to $1 \mathrm{e}-4$.

\subsection{Choice of Planes and Points}

Figure 2 shows the choice of planes (from sections 1-1 to 7-7 are denoted as Cs 1 and Cs7 correspondingly) across the inlet pipe along with the mean streamline starts from entry $(x / l=0)$ to the exit $(x / l=1)$ of inlet pipe. The choice of monitoring points and locations will be helpful to compare with the experimental findings in later stage. 


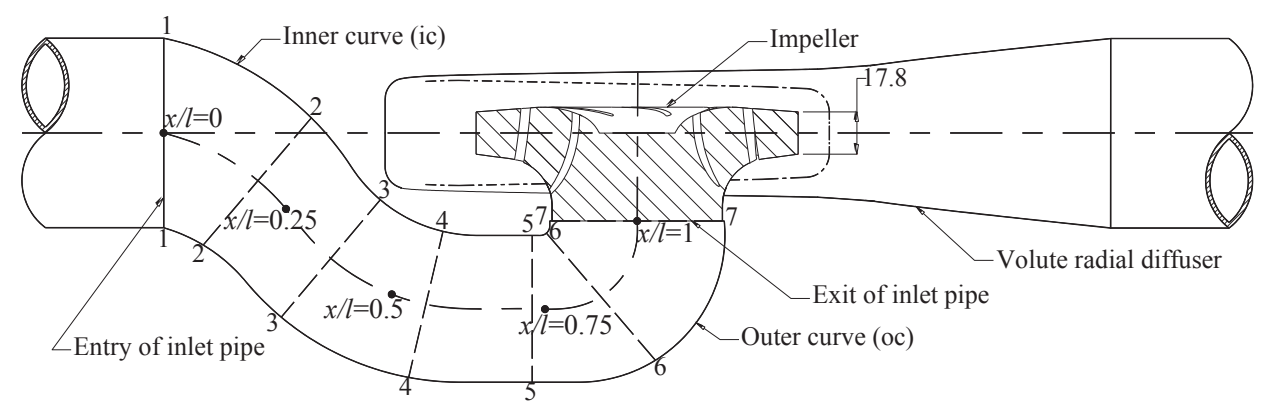

Fig.2. Sketch of vertical inline pump

\section{Experimental Test Rig}

The open test rig was used to conduct the performance characteristics of vertical inline pump from shut off to full open conditions of throttle valve. The pressure at the suction and delivery sides of the pump was measured with the help of pressure transmitter in the range of 0-1.6 bar (absolute) and 0-4 bar respectively. The entire tests were conducted at constant speed and it was controlled using variable frequency drive. The input power to the motor was measured through Wattmeter and the flow rate through pump was obtained with the help of electromagnetic flow meter. The uncertainty of measurement for the total head, flow rate, input power, and efficiency are within the allowable limits. The experiments were repeated to get the repetition of characteristics.

\section{Results and Discussion}

\subsection{Performance characteristics of pump}

The hydraulic characteristics using experimental (exp) readings along with the computational (cfd) results are plotted in Fig. 3 in non dimensional form. The total head, flow rate, and input power are given in terms of head $(\psi)$, flow $(\phi)$, and power $(v)$ coefficients respectively. The best efficiency (bep) of the pump during testing is found to be $72.5 \%$, but the hydraulic efficiency from the cfd is about $82 \%$ for the same flow with the difference in head coefficient between the simulation and the experiment is $3.8 \%$. The agreement between head coefficient is found be well near the best efficiency point as well near low flow rate zone, whereas the deviation is found at higher flow rates. The reason for deviation is due to hydraulic head losses inside the impeller, in addition to shock losses at the entry of impeller. Based on head and power coefficients, the efficiency curves are drawn and found to have more deviation between experiment and computational values. Experimental efficiency is nothing but overall efficiency of the pump, which includes leakage losses, friction losses inside the pump, mechanical losses, and so on.

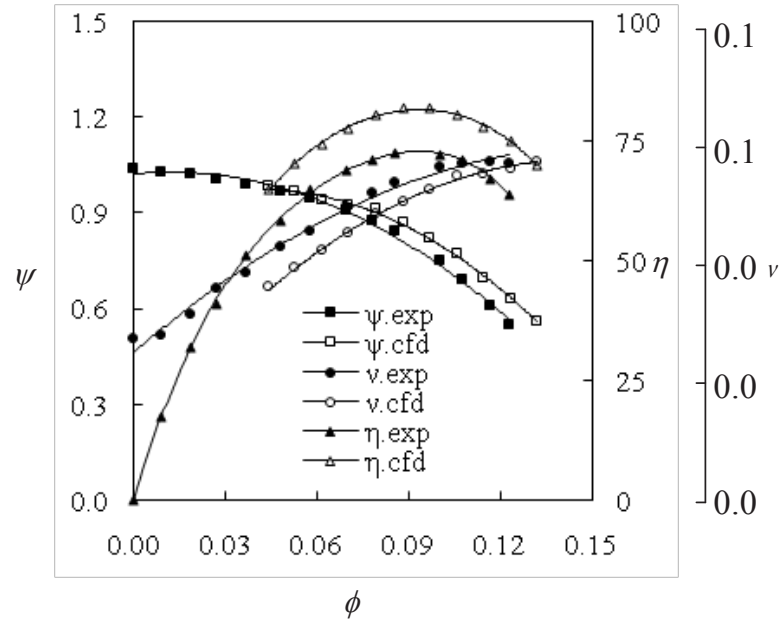

Fig.3. Performance characteristics of pump

\subsection{Sound power level}

Another benchmark for the computational results is with respect to sound power level $\left(L_{W A}\right)$ obtained from the pump. Fritz et al. [19] have given a relation to found out the total A-weighted sound power level from centrifugal pump based on the input power of motor, which includes the pipelines. Similarly Schmitt and Klein [20], used to calculate the same sound power for pump (alone) and another relation to calculate the magnitude without the influence of motor and gear box [21]. The sound power level from computational results is compared with empirical formula [19-21], which are given in Table 1 and Table 2.

Table 1. Sound power level from empirical relation

\begin{tabular}{ccc}
\hline Input power $(\mathrm{kW})$ & Reference & $L_{W A}(\mathrm{~dB})$ \\
\hline 3.45 & {$[27]$} & 76.6 \\
(at BEP) & {$[28]$} & 66.5 \\
& {$[29]$} & 77.8 \\
\hline 3.58 & {$[27]$} & 76.9 \\
(at full open) & {$[28]$} & 66.6 \\
& {$[29]$} & 78.0 \\
\hline
\end{tabular}

\footnotetext{
Corresponding author: christo_spc@yahoo.com
} 
Table 2. Sound power level from computation

\begin{tabular}{ccc}
\hline Harmonics & $\begin{array}{c}\text { frequency } \\
(\mathrm{Hz})\end{array}$ & $\begin{array}{c}L_{W A} \\
(\mathrm{~dB})\end{array}$ \\
\hline $1^{\text {st }}(\mathrm{fs})$ & 48.5 & 71.8 \\
$6^{\text {th }}(\mathrm{vpf})$ & 291.0 & 53.1 \\
\hline
\end{tabular}

The magnitudes are calculated based on best efficiency point (BEP) and maximum input power for vertical inline pump at design speed of non cavitating condition with reference to $1 \mathrm{~kW}$ power. From computation, the sound power level is calculated and found to be function of observer radius $(1 \mathrm{~m})$, acoustic pressure, acoustic impedance, and the acoustic reference pressure in logarithmic form. Table 2 shows the magnitude of sound power level at shaft frequency (fs) and vane passing frequency (vpf). The variation in magnitude of sound power with respect to empirical equations is about $6 \%-8 \%$ higher with reference to [27,29] and less than $8 \%$ for [28] with respect to first harmonics (i.e., shaft frequency). This confirms that the prediction of sound power level from numerical tool is well agreeable and gives the scope for further investigation of acoustics in flow domains.

\subsection{Velocity coefficient distribution}

Figure 4 shows the streamline of velocity coefficient $(\mathrm{Cv})$ for inlet pipe and impeller (meridional view) at $0.5 \mathrm{Qn}, \mathrm{Qn}$, and $1.5 \mathrm{Qn}$. Around 30 points of equally spaced 3D streamlines are used to represent the flow stream along the inlet pipe with the gray background for clear visibility and represented in 2D form. It is inferred that the flow disturbance is more for the case of $0.5 \mathrm{Qn}$ than compared with Qn and 1.5Qn. It is due to the recirculation and return flow effects from the impeller and similar phenomenon were visualized by Posa et al. [22] and found to extend to the upstream of impeller. Due to this effects, noise due to turbulence increases, when flow is less than Qn. In addition, the flow passage of inlet pipe is not well designed even for the case of nominal flow rate; hence there is scope for optimizing the inlet pipe for this pump. All the streamlines and contours are given for the same time step $(30.9 \mathrm{~ms})$ for comparison.
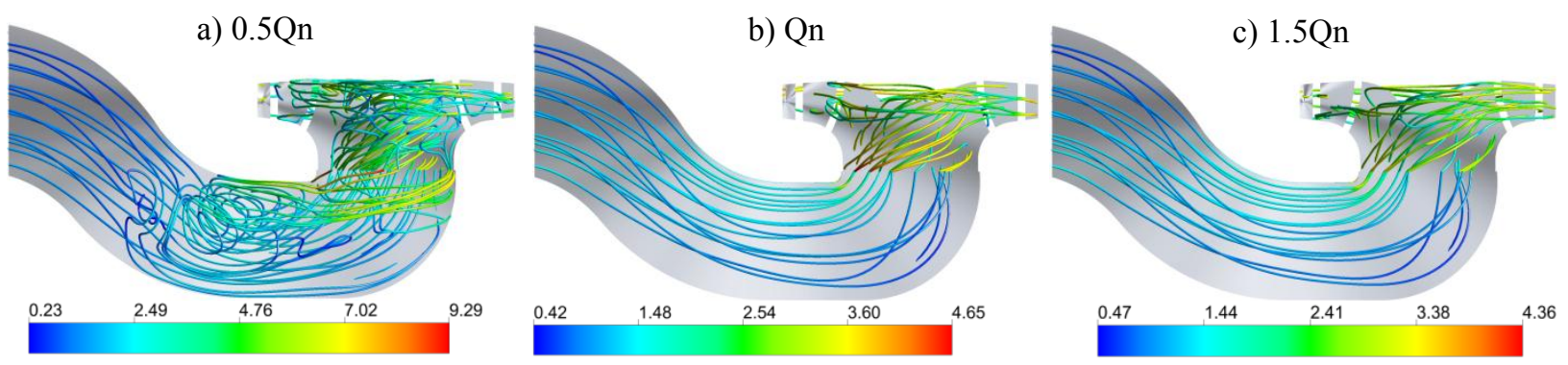

Fig. 4. Streamlines of velocity coefficient

More details about the sound source level across each cross sections of inlet pipe were discussed for 0.5Qn in the following section 4.4. In general, the flow through bends and elbow are very complex because the pressure gradients changes drastically from one to another locations, which results in increase of turbulence intensity and hence leads to flow induced noise and structural vibration. Hambric et al. [23] discovered the similar kind of phenomenon like secondary flow fields because of strong pressure gradients at the downstream of elbow. This confirms the possibility of noise generation from the turbulence in inlet pipe of vertical inline pump.

\subsection{Source of noise across cross sections at $0.5 \mathrm{Qn}$}

The dipole (DSL) and quadrupole (QSL) sources in Eq. (1) is rewritten in normalized form as the ratio of corresponding source terms with respect to acoustic reference pressure for water $(1 \mu \mathrm{Pa})$ and were calculated on the near field. As stated in section 2.1, the dipole source is directly proportional to fluctuation in pressure; hence the contours of dipole are similar to pressure contours. Figure 5 and 6, shows the contours of DSL,
QSL, and Psp for the cross sections across the inlet pipe at $0.5 \mathrm{Qn}$. From DSL contours, it shows that the zone of high and low pressure varies from one to other sections drastically. This creates a way for recirculation of flow inside the inlet pipe apart from the reduction of flow rate in pump. The monopole source is neglected, since its significance is low when compared with other two sources in stationary flow domain. The top of each section represents the point on inner curve (ic) for Fig. 5 $\& 6$. The zone of low pressure is evident at the bottom of Cs2 and moves in clockwise direction until Cs5 (see Fig. 6), whereas no distinct low pressure is found in Cs6.

From $\mathrm{Cs} 3$ to $\mathrm{Cs} 7$, the uniformity of pressure pattern is not observed; it is due to reduced flow rate. A QSL contour is nothing but the indication of turbulence or vortices in the flow field. When compared with DSL, the magnitude of QSL is small, but it should not be neglected in order to have an optimized design of any flow passage. It is found that there is no direct correlation between DSL and QSL for all cross sections of inlet pipe. Each section is having its own turbulence intensity, but it is not sufficient enough to create noise. A only few proportions from fluctuating turbulence power are utilized to generate sound power [24]. 


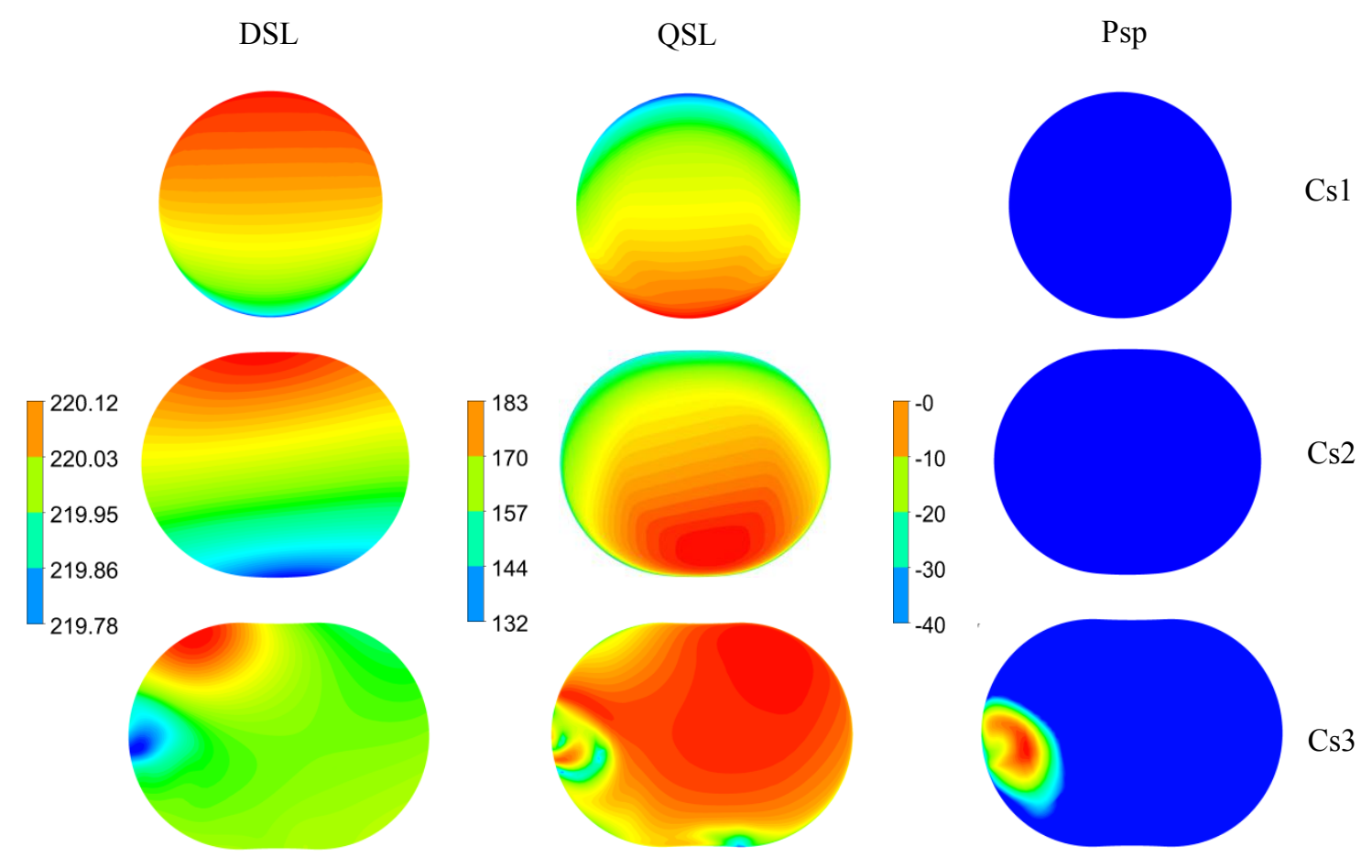

Fig. 5. Contours of DSL, QSL, and Psp at $0.5 \mathrm{Qn}$ for Cs1, Cs2, and Cs3
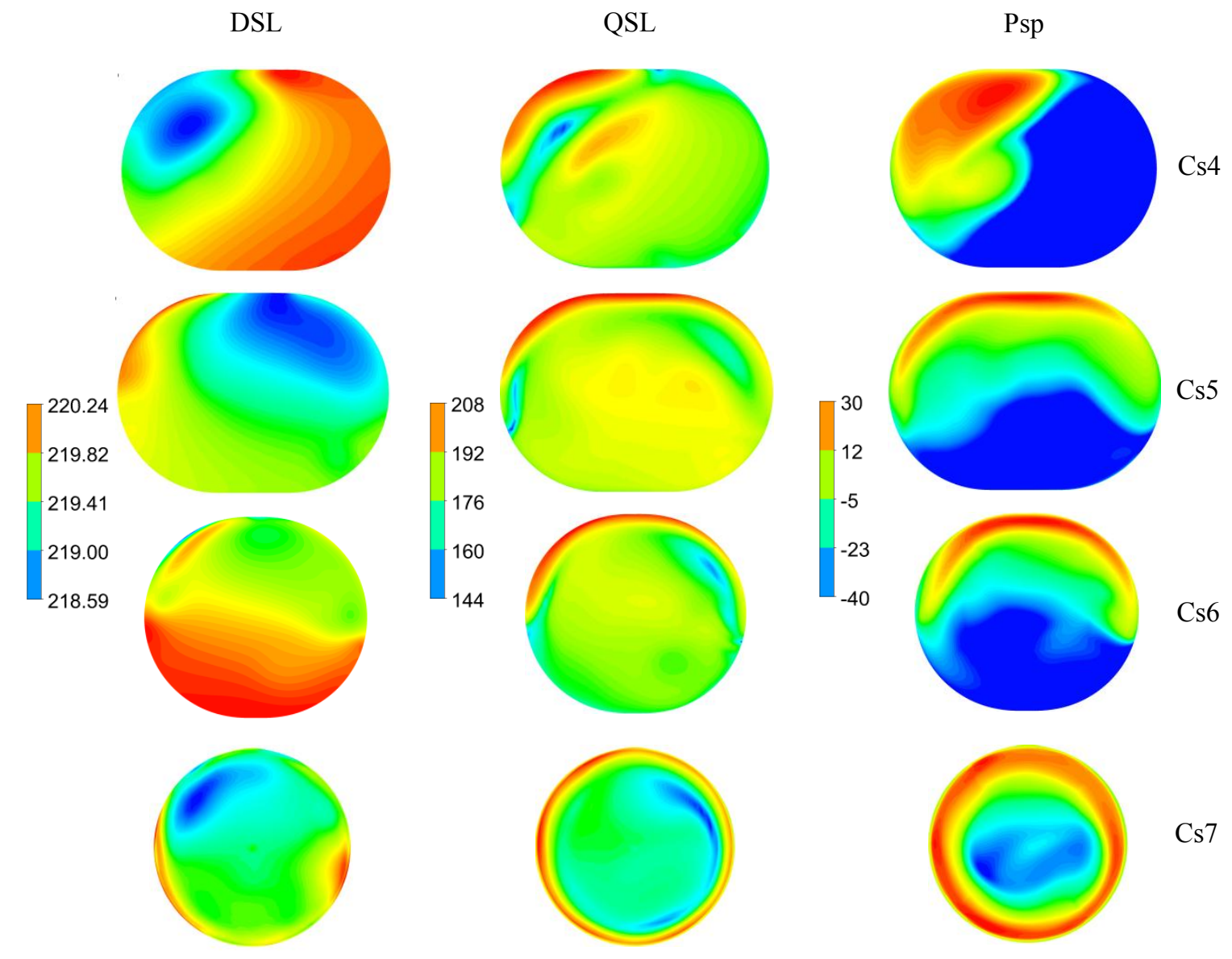

Fig. 6. Contours of DSL, QSL, and Psp at 0.5 Qn from Cs4 to Cs7 
Proudman sound power level (Psp) for Cs1 and Cs2 are $-40 \mathrm{~dB}$, which means that the turbulence is very minimal (i.e., zero). From Cs3, the intensity level of vortices increases drastically, till it reaches the exit of inlet pipe (i.e., Cs7). Psp starts from Cs3 at left hand side of section and increases in Cs4 at top position of left hand side, whereas for Cs5 and Cs6, the entire top surfaces are filled with turbulence phenomena. This may be due to sudden curvature (or short radius) of inlet pipe and also it confirms the swirl flow due to the pressure gradients and operating condition of pump. In Cs7, the intensity of vortices is observed around the periphery of cross sections and minimal at the core, this ensure the helicity (product of vorticity and velocity) at that section [25]. Even for end suction centrifugal pump, Fu et al. [26] confirmed the presence of low frequency oscillation of inlet pressure near the impeller eye by means of fast response pressure transducers. Similar kind of cross sectional studies can be extended to other flow rates for more detailing of inlet pipe flow passage.

\section{CONCLUSIONS}

The flow induced noise in vertical inline pump is studied using Lighthill analogy for various flow rates and examined for inlet pipe domain. Experimental characteristics of pump are well matched with computational readings and slight variation is found at off-design conditions, it may be due to the losses in pump. The comparison between empirical relations suggested by various researchers for sound power level with the computed values shows that it agrees well with the magnitude at shaft frequency. From velocity streamlines along the inlet pipe it infers that the intensity of turbulence was more for lower flow rates and less for higher flow rates. But due to existing curvature in inlet pipe, the turbulence exists even for other flow rates. The same is confirmed from cross sections contours of quadrupole source and Proudman sound power levels. This study enables the pump user to optimize the shape of flow domain with respect to flow induced noise, but proper balance as to be adapted between hydraulic, structural and acoustic aspects.

\section{Acknowledgment}

This work was supported by the state key program of National Natural Science Funds of China (Grant No. 51239005).

\section{Nomenclature}

$A_{2} \quad=\pi d_{2} b_{2}$ outlet area of impeller, $\mathrm{m}^{2}$

$b_{2} \quad$ outlet width of impeller, $\mathrm{m}$

$c_{s} \quad$ speed of sound in working fluid, $\mathrm{m} \mathrm{s}^{-1}$

$\mathrm{Cv} \quad=V_{c s} / V_{\text {enip }}$ velocity coefficient, non-dimensional

$d_{2} \quad$ outlet diameter of impeller, $m$

DSL $=20 \log \left(\right.$ dipole source term $\left./ P_{\text {Arefpr }}\right)$ dipole sound level, $\mathrm{dB}$ re $1 \mu \mathrm{Pa}$

$f \quad$ non-zero at fluid region, and zero at the moving boundary

$g \quad$ acceleration due to gravity, $\mathrm{m} \mathrm{s}^{-2}$

$H \quad$ total head, $\mathrm{m}$

$k \quad$ turbulent kinetic energy, $\mathrm{m}^{2} \mathrm{~s}^{-2}$

$l$ total length of mean streamline in inlet pipe, $\mathrm{m}$

$L_{W A} \quad$ sound power level, $\mathrm{dB}$ re $1 \mathrm{~kW}$

$n \quad$ rotational speed, $\mathrm{rpm}$

$n_{S} \quad=n \sqrt{Q} / H^{3 / 4}$ metric specific speed

$P_{A} \quad$ Proudman sound power, $\mathrm{W} \mathrm{m} \mathrm{m}^{-3}$

Psp $=10 \log \left(P_{A} / P_{\text {Arefpo }}\right)$ Proudman sound power, $\mathrm{dB}$ re $10^{-12} \mathrm{~W} \mathrm{~m}^{-3}$

$P_{\text {Arefpo }} \quad$ acoustic reference power, $10^{-12} \mathrm{~W} \mathrm{~m}^{-3}$

$P_{\text {Arefpr }} \quad$ acoustic reference pressure, $\mathrm{Pa}$

$Q \quad$ flow rate, $\mathrm{m}^{3} \mathrm{~s}^{-1}$

$Q_{n} \quad$ nominal flow rate, $\mathrm{m}^{3} \mathrm{~s}^{-1}$

QSL $=20 \log \left(\right.$ quadrupole source term $\left./ P_{\text {Arefpr }}\right)$ quadrupole sound level, $\mathrm{dB}$ re $1 \mu \mathrm{Pa}$

$t \quad$ time, $\mathrm{s}$

$T_{i j} \quad$ momentum flux tensor, $\mathrm{N} \mathrm{m}^{-2}$

$u_{2} \quad=\pi d_{2} n / 60$ peripheral velocity at outlet of impeller, $\mathrm{m} \mathrm{s}^{-1}$

$v_{n} \quad$ normal velocity of the surface, $\mathrm{m} \mathrm{s}^{-1}$

Corresponding author: christo_spc@yahoo.com 


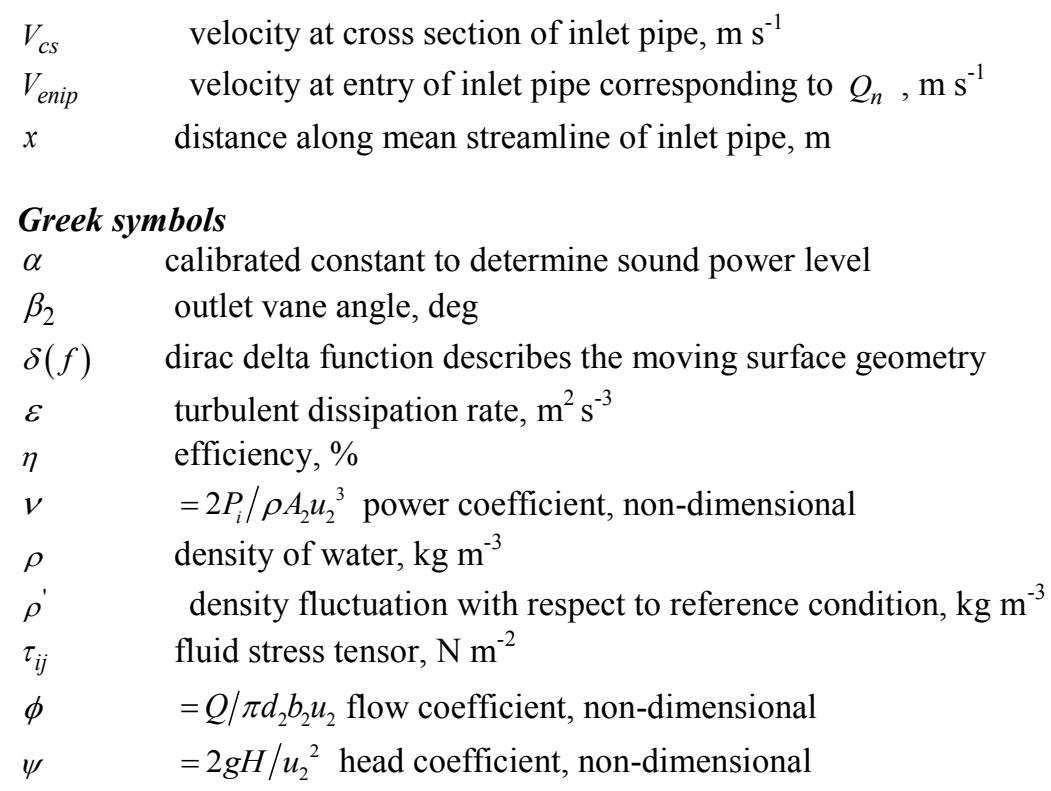

\section{Reference}

1. J. F. Gulich, Centrifugal pumps (third ed., Springer, New York, 2014)

2. H. C. Simpson, R. Macaskill, T. A. Clark, Proc. of the Institution of Mech. Engineers, Conference Proc., 181, 84-108 (1966)

3. R. Dong, S. Chu, J. Katz, J. Turbomachinery. 119, 506-515 (1997)

4. M. S. Howe, Acoustics of fluid-structure interaction (Cambridge Univ. Press, Cambridge, 1998)

5. M. A Langthjem, N. Olhoff, J. Fluids and Struc. 19, 369-386 (2004)

6. Y. Y. Jiang, S. Yoshimura, R. Imai, H. Katsura, T. Yoshida, C. Kato, J. Fluids and Struc. 23, 531-544 (2007)

7. J. Huang, S. Geng, R. Wu, K. Liu, C. Nie, H. Zhang, Acta Acoust. 35, 113-118 (2010)

8. X. Yong, W. Chunlin, Y. Xiaoyong, Y. Jian, Z. Cheng, Chinese J. Drain. and Irri. Mach. Eng. 34, 483-489 (2016)

9. M. Gao, P. Dong, S. Lei, A. Turan, Energies. 221, 1-11 (2017)

10. M. Lighthill, Proc. of the Royal Society $A$, Mathematical, Physical and Eng. Sci. 211, 564-587 (1952)

11. J. E. F. Williams, D.L. Hawkings, Proc. of the Royal Society A, Mathematical, Physical and Eng. Sci. 264, 321-342 (1969)

12. J. E. F. Williams, D.L. Hawkings, J. Sound and Vib. 10, 10-21 (1969)

13. K. R. Fritz, C. C. Hantschk, S. Heim, H. Nürnberger, E. Schorer, B. Stüber, D. Vortmeyer, Flow noise, in G. Muller, M. Moser (Eds.), (Springer-Verlag Berlin Heidelberg, 2013)
14. D. B. Hanson, M. R. Fink, J. Sound and Vibration. 62, 19-38 (1979)

15. I. Proudman, Proc. of the Royal Society A, Mathematical, Physical and Eng. Sci. 214, 119-132 (1952)

16. S. Sarkar, M.Y. Hussaini, NASA Contract Report 93-74, NASA Langley Research Center, Hampton, Virginia. 1993.

17. B. Lakshminarayana, J. Fluids Eng. 113, 315-352 (1991)

18. ANSYS, ANSYS CFX User's Guide, Release 17.0, (ANSYS, Inc., Canonsburg, PA, 2017)

19. B. Stüber, K. Fritz, F. Lang, Stand der technik bei der lärmminderung inder petrochemie, (Umweltbundesamt, Berlin, 1981)

20. A. Schmitt, V. Klein, Schallemission von chemienormpumpen, (Chemie-Anlagen und Verfahren 6, 1979)

21. Europump leitfaden, (Geräuschemission bei kreiselpumpen, 2002)

22. A. Posa, A. Lippolis, E. Balaras, J. Fluids Eng. 138, 121101-1-121101-13 (2016)

23. S. A. Hambric, D. A. Boger, J. B. Fahnline, R.L. Campbell, J. Fluids and Struc. 26, 121-147 (2010)

24. B. A. De Jong, M.S Thesis, Faculty of Mech. Eng., (Delft Univ. of Tech., 2016)

25. S. Christopher, S. Yuan, J. Pei, G.X. Cheng., J. Fluids Eng. 140, 051201-1-051201-10 (2017)

26. Y. Fu, J. Yuan, S. Yuan, G. Pace, L. d'Agostino, P. Huang, X. Li, J. Fluids Eng. 137, 011102-1011102-12 (2015) 